\title{
Looking at the Other Side of the Coin: Is the Cerebellum Involved in Sudden Unexpected Death in Mitochondrial Disorders?

\author{
Fulvio A. Scorza ${ }^{1}$, Ana C. Fiorini' ${ }^{2}$, Carla A. Scorza ${ }^{1}$, Josef Finsterer ${ }^{3}$ \\ ${ }^{1}$ Disciplina de Neurociência. Escola Paulista de Medicina/Universidade Federal de São Paulo/. (EPM/ \\ UNIFESP). São Paulo, Brasil. \\ ${ }^{2}$ Programa de Estudos Pós-Graduado em Fonoaudiologia, Pontifícia Universidade Católica de São Paulo (PUC-SP). \\ Departamento de Fonoaudiologia, Escola Paulista de Medicina/Universidade Federal de São Paulo (EPM/ \\ UNIFESP). São Paulo, Brasil. \\ ${ }^{3}$ Krankenanstalt Rudolfstiftung, Vienna, Austria. \\ fifigs1@yahoo.de
} \\ *Corresponding Author: Finsterer J, MD, PhD, Krankenanstalt Rudolfstiftung, Vienna, Austria, Europe.
}

\section{LETTER TO THE EDITOR}

We read with great interest the stimulating article by Schreglmann et al. who showed that isolated dystonia so far classified as idiopathic can be a manifestation of a mitochondrial disorder (MID), thus strengthening the link between cerebellar involvement and movement disorders in MIDs ${ }^{1}$. According to these interesting results and new research perspectives on the subject, the cerebellum can be prominently affected in MIDs. In this context, macro-evolutionary studies of the cerebellum contributed to theories on cerebellar functions and connectivity, providing new insights in the evolutionary pathways that underlie cerebellar functions ${ }^{2}$. Furthermore, recent neuroimaging findings demonstrated that the cerebellum is involved in autonomic regulations ${ }^{3}$. Briefly, it was shown that the cerebellum coordinates vestibular stimuli and blood pressure changes, and that cerebellar injury results in substantially altered timing of responses to pressor challenges in sleep-disordered breathing conditions ${ }^{3}$. Interestingly, several cerebellar pathological findings are reported in the sudden infant death syndrome (SIDS) and it has been proposed that cerebellar atrophy may lead to an inability to recover from apnea or apneusis, or failure to recover from extreme hypotension or arrhythmia, ultimately resulting in sudden death in patients with refractory epilepsy4. Following this line of reasoning, sudden death in MIDs is well described in the literature and sudden cardiac death (SCD) is an important cause of death in MIDs necessitating the identification of risk factors for SCD in MIDs by appropriate management ${ }^{5}$. In addition, Eom and colleagues investigated the clinical characteristics of risk factors for death in pediatric MID patients ${ }^{6}$. The authors showed that 29\% of their MID patients died suddenly at $<6$ years of age and that early death was associated with lesions in the thalamus and the number of organs involved in the MID ${ }^{6}$. Considering these results together, it is reasonable to establish a connection between cerebellar abnormalities and increased mortality and even sudden death in MID patients.

Overall, there is a need for further studies and a need to establish first hypotheses about the pathogenesis of these phenomena. While answers to the many of the open questions are not extensively discussed in the literature, caution in the management of MID patients is truly necessary.

\section{ACKNOWLEDGEMENTS}

Our studies are supported by the following grants: FAPESP (Fundação de Amparo à Pesquisa do Estado de São Paulo); CNPq (Conselho Nacional de Desenvolvimento Científico e Tecnológico); Coordenação de Aperfeiçoamento de Pessoal de Nível Superior (CAPES) and FAPESP/CNPq/MCT (Instituto Nacional de Neurociência Translacional). 
Looking at the Other Side of the Coin: Is the Cerebellum Involved in Sudden Unexpected Death in Mitochondrial Disorders?

\section{REFERENCES}

[1] Schreglmann SR, Riederer F, Galovic M et al. Movement disorders in genetically confirmed mitochondrial disease and the putative role of the cerebellum. Mov Disord. 2018; 33(1): 146-155.

[2] Smaers JB. Modeling the evolution of the cerebellum: from macroevolution to function. Prog Brain Res. 2014; 210: 193-216.

[3] Macey PM, Ogren JA, Kumar R, Harper RM. Functional Imaging of Autonomic Regulation: Methods and Key Findings. Front Neurosci. 2016; 9: 513.
[4] Scorza FA, Terra VC, Arida RM, Sakamoto AC, Harper RM. Sudden death in a child with epilepsy: potential cerebellar mechanisms? Arq Neuropsiquiatr. 2011; 69(4): 707-10.

[5] Finsterer J, Stöllberger C, Maeztu C. Sudden cardiac death in neuromuscular disorders. Int J Cardiol. 2016; 203: 508-15.

[6] Eom S, Lee HN, Lee S, Kang HC, Lee JS, Kim HD, Lee YM. Cause of Death in Children With Mitochondrial Diseases. Pediatr Neurol. 2017; 66: 82-88.

Citation: Fulvio A. Scorza, Ana C. Fiorini, Carla A. Scorza, Josef Finsterer. Looking at the Other Side of the Coin: Is the Cerebellum Involved in Sudden Unexpected Death in Mitochondrial Disorders?. Open Access Journal of Internal Medicine. 2019; 2(1): 49-50.

Copyright: (C) 2019 Fulvio A. Scorza, Ana C. Fiorini, Carla A. Scorza, Josef Finsterer. This is an open access article distributed under the Creative Commons Attribution License, which permits unrestricted use, distribution, and reproduction in any medium, provided the original work is properly cited. 